\title{
Tin Whisker Electrical Short Circuit Characteristics-Part II
}

\author{
Karim J. Courey, Shihab S. Asfour, Arzu Onar, Jon A. Bayliss, Lawrence L. Ludwig, and Maria C. Wright
}

\begin{abstract}
Existing risk simulations make the assumption that when a free tin whisker has bridged two adjacent exposed electrical conductors, the result is an electrical short circuit. This conservative assumption is made because shorting is a random event that has an unknown probability associated with it. Note however that due to contact resistance, electrical shorts may not occur at lower voltage levels. In our first paper, we developed an empirical probability model for tin whisker shorting. In this paper, we develop a more comprehensive empirical model using a refined experiment with a larger sample size, in which we studied the effect of varying voltage on the breakdown of the contact resistance which leads to a short circuit. From the resulting data, we estimated the probability distribution of an electrical short, as a function of voltage. In addition, the unexpected polycrystalline structure seen in the focused ion beam (FIB) cross section in the first experiment was confirmed in this experiment using transmission electron microscopy (TEM). The FIB was also used to cross section two card guides to facilitate the measurement of the grain size of each card guide's tin plating to determine its finish.
\end{abstract}

Index Terms-Contact resistance, focused ion beam (FIB), short circuit, tin whiskers, transmission electron microscopy (TEM).

\section{INTRODUCTION}

$\mathbf{T}$ IN WHISKERS are crystalline filamentary surface eruptions from a tin-plated surface that can have a variety of shapes including straight, kinked, and curved [1]. The hazards presented by tin whiskers include temporary and permanent electrical short circuits, debris contamination, and metal vapor arcing [2]. Failures attributed to metal whiskers have been documented in many industries including the nuclear power,

Manuscript received July 31, 2008; revised October 28, 2008. Current version published January 08,2009 . This document was prepared under the sponsorship of the National Aeronautics and Space Administration. Neither the United States government nor any person acting on behalf of the United States government assumes any liability resulting from the use of the information contained in this document, or warrants that such use will be free from privately owned rights. This work was supported in part by S. Stich, S. M. Poulos, A. Oliu, J. N. Cowart, and S. J. McDanels of the National Aeronautics and Space Administration. This work was recommended for publication by Associate Editor R. Gedney upon evaluation of the reviewers comments.

K. J. Courey is with the Orbiter Sustaining Engineering Office, National Aeronautics and Space Administration, Kennedy Space Center, FL 32899 USA (e-mail: karim.j.courey@nasa.gov).

S. S. Asfour is with the College of Engineering, University of Miami, Coral Gables, FL 33146 USA (e-mail: sasfour@miami.edu).

A. Onar is with the Biostatistics Department, St. Jude Children's Research Hospital, Memphis, TN 38105 USA (e-mail: arzu.onar@stjude.org).

J. A. Bayliss, L. L. Ludwig, and M. C. Wright are with the Materials Science Division, National Aeronautics and Space Administration, Kennedy Space Center, FL 32899 USA (e-mail: jon.a.bayliss@nasa.gov; lawrence.1.ludwig@nasa.gov; m.clara.wright@nasa.gov).

Color versions of one or more of the figures in this paper are available online at http://ieeexplore.ieee.org.

Digital Object Identifier 10.1109/TEPM.2008.2009224 computer, satellite, missile, military aircraft, and medical industries [3]. An extensive list of metal whisker failures and extensive documentation of the tin whisker phenomena can be found on the National Aeronautics and Space Administration (NASA) Goddard Space Flight Center tin whisker home page (http://nepp.nasa.gov/WHISKER/).

Tin whiskers can pose serious problems in high-reliability systems that can result in loss of life as well as significant capital losses. Improving our ability to assess the risk associated with tin whiskers is an important area of study for both the government and private industry [4].

Risk simulations have been developed by the Center for Advanced Life Cycle Engineering (CALCE) at the University of Maryland, and TYCO Electronics [5], [6] . In these simulations, it is assumed that physical contact between a whisker and an exposed contact results in an electrical short. This conservative assumption was made because the probability of an electrical short from tin whiskers had not yet been determined at the time the simulations were written [4]. Fang [5] noted the difference between experiment and simulated results and attributed the difference to contact resistance.

The voltage level at the transition to metallic conduction current is the voltage level at which the contact resistance breaks down. Experiments were designed to develop an empirical model to quantify the probability of occurrence of an electrical short circuit from tin whiskers as a function of voltage. In our first paper, we developed an empirical probability model for tin whisker shorting [4].

The second experiment which is detailed in this paper incorporated the lessons learned from the first experiment. It determined the breakdown voltage for a larger sample $(n=200$ versus $n=35$ ) of whiskers with the objective of obtaining a more comprehensive empirical distribution. The observed data was used to determine the best fitting parametric distribution to the breakdown voltage. The associated data analysis also incorporated explorations of possible sources of variability. In addition, transmission electron microscopy (TEM) was used to study and determine if the whisker that was sectioned in the first experiment was polycrystalline or a single crystal. Also, an FIB was used to section a sample of one card guide from each ascent thrust vector controller (ATVC) to determine if the plating that grew the whiskers was bright or matte finish.

\section{BACKGROUND}

In our first experiment, we found the best fitting distribution was the three parameter (3P)-inverse Gaussian (IG) distribution [4]. The parameters for the 3P-IG Distribution were 


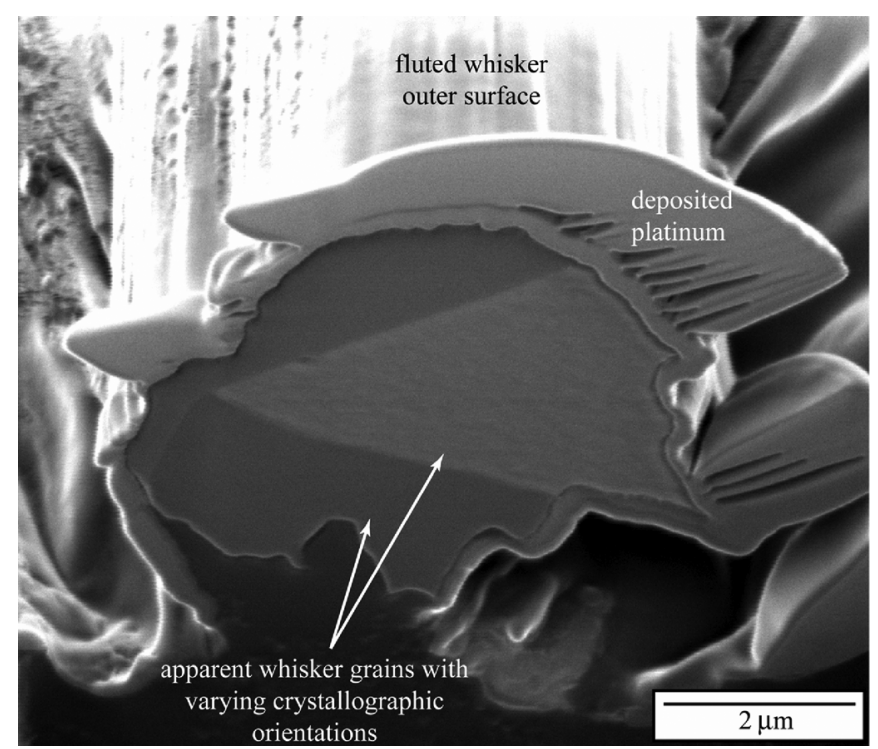

Fig. 1. FIB image of as-sectioned tin whisker shows apparent variation in grain orientation within the cross section. The image was taken at a $52^{\circ}$ angle from horizontal [NASA/University of Central Florida (UCF)]. Reproduced from [4].

$\lambda=31.977, \mu=17.571, \gamma=-1.9716$. The probability density function (pdf) for the $3 \mathrm{P}-\mathrm{IG}$ distribution is shown as follows [7]:

$$
f(x)=\sqrt{\frac{\lambda}{2 \pi(x-\gamma)^{3}}} \exp \left(-\frac{\lambda(x-\gamma-\mu)^{2}}{2 \mu^{2}(x-\gamma)}\right) .
$$

Based on our data, the expected voltage (mean) where a short will occur for the 3P-IG distribution is $\mu+\gamma=15.5994 \mathrm{Vdc}$, with a variance of $\mu^{3} / \lambda=169.6491$ [8]. Tin whiskers from the same card guide used in the breakdown voltage experiment were cross sectioned using an FEI 200 TEM FIB with a 30-kV Gallium liquid metal ion source. The ion beam was used to mill away whisker material until the desired region of interest was exposed to obtain a cross section normal to the whisker's growth direction [4].

The FIB cross section facilitated the examination of what appeared to be grains with varying crystallographic orientations within the tin whisker as illustrated in Fig. 1. An additional two whiskers from the card guide were removed and sectioned by the FIB. These smaller diameter whiskers exhibited the commonly reported single crystal structure.

\section{EXPERIMENTAL PROCEDURES}

\section{A. Tin Whisker Breakdown Voltage Experiment}

To determine the break down voltage, a micromanipulator probe was brought in contact with the side of a tin whisker growing from a tin-plated beryllium copper card guides as illustrated in Fig. 2.

The cantilever beam whisker model shown in Fig. 3 from our first paper illustrates the mechanical load placed on the whisker by the micromanipulator probe [4].

In this model, $P=$ the force applied to the whisker, $L=$ the distance from the base of the whisker to the applied force,

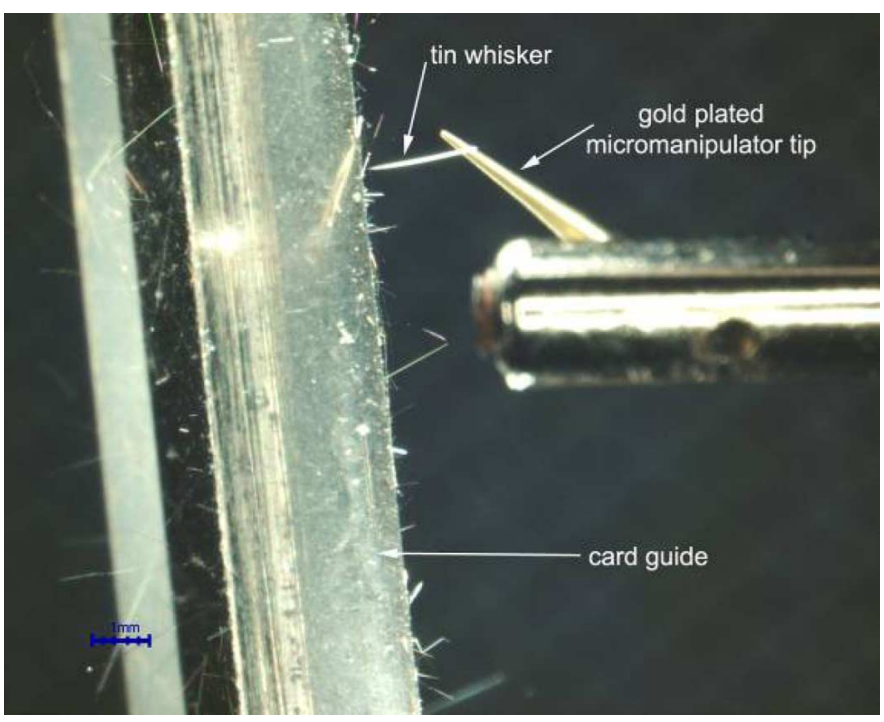

Fig. 2. Micromanipulator probe in contact with a tin whisker.

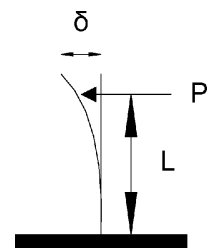

Fig. 3. Cantilever beam whisker model. Reproduced from [4].

$\delta=$ the whisker deflection, $I=$ the moment of inertia, and $E=$ the modulus of elasticity [4].

$$
P=\frac{3 E I \delta}{L^{3}} \text {. }
$$

From (1.2), the distance from the base of the whisker to the applied force has a much greater effect on the force applied to the whisker than the whisker deflection. Since our objective was to minimize the force applied to the whisker, the micromanipulator probe was applied to approximately the top $25 \%$ of the length of the whisker. In addition, as soon as contact was made between the micromanipulator probe and the whisker, as seen under a microscope, the probe was not moved any further. This minimized whisker deflection. The combination of these two controls minimizes the force applied to the whisker.

Data acquisition software was written using LabVIEW to automate both the incrementing of power supply voltage changes as well as the gathering of the voltage and current data for each of the tin whiskers. The schematic diagram of the test station is shown in Fig. 4. Once contact was established, as determined with an optical microscope, the power supply voltage was increased from 0 to $45 \mathrm{Vdc}$ in $0.1-\mathrm{Vdc}$ increments [4]. This was the same software used in the first experiment.

The software captured 3 to 4 samples per second over the entire voltage range. The automated test fixture was validated by substituting a calibrated resistor decade box for the micromanipulator, whisker, and card guide. The experiment was repeated to develop an empirical probability distribution of shorting as a function of voltage [4]. 


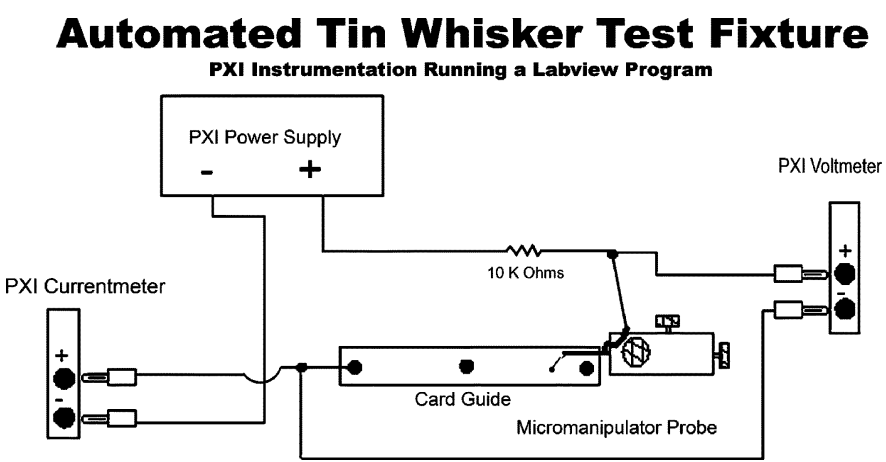

Fig. 4. Schematic diagram for the tin whisker test station instrumentation. Reproduced from [4].

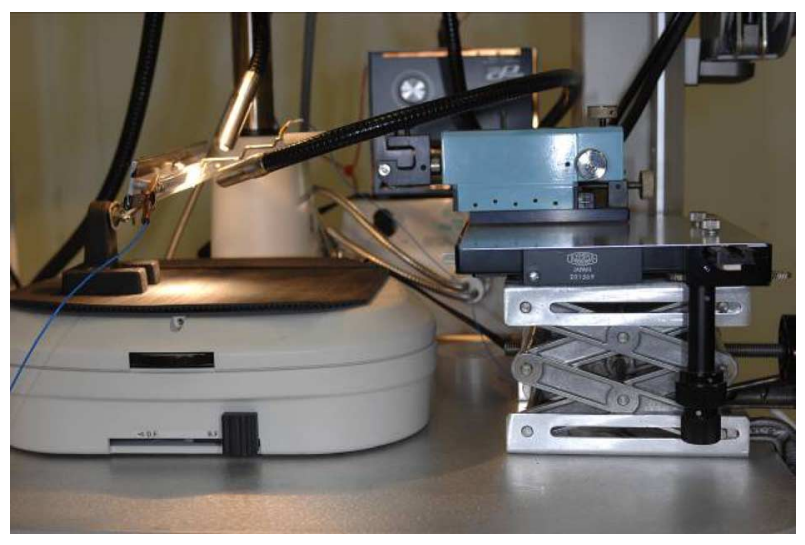

Fig. 5. Tin whisker test station probing area close up.

\section{B. Improvements to the Initial Experiment}

The following improvements were added to this experiment.

- Improved electrical grounding by connecting to the card guide instead of the card holding fixture.

- Gold-plated tungsten micromanipulator tips were used to minimize the effect of any oxides on the probe.

- The probe tip was cleaned every five whiskers with isopropyl alcohol.

- A solderer's helper was modified to allow flexible positioning of the card guide and an extension platform for the microscope was fabricated to facilitate clamping of the lab jack (refer to Fig. 5).

- A ferrous top plate was fabricated for the lab jack to allow the magnetic base of the micromanipulator to be firmly mounted on the lab jack. The lab jack provided the coarse $X, Y$, and $Z$ movements, while the micromanipulator provided the fine $X, Y$, and $Z$ movements for probing the tin whiskers (refer to Fig. 5).

\section{Method of Sample Selection}

In the first experiment, all 35 whiskers tested were from the same card guide. In this experiment, card guides from two different line replaceable units (LRUs) were available for sampling. The card guides are from ATVC Serial Number 31 and

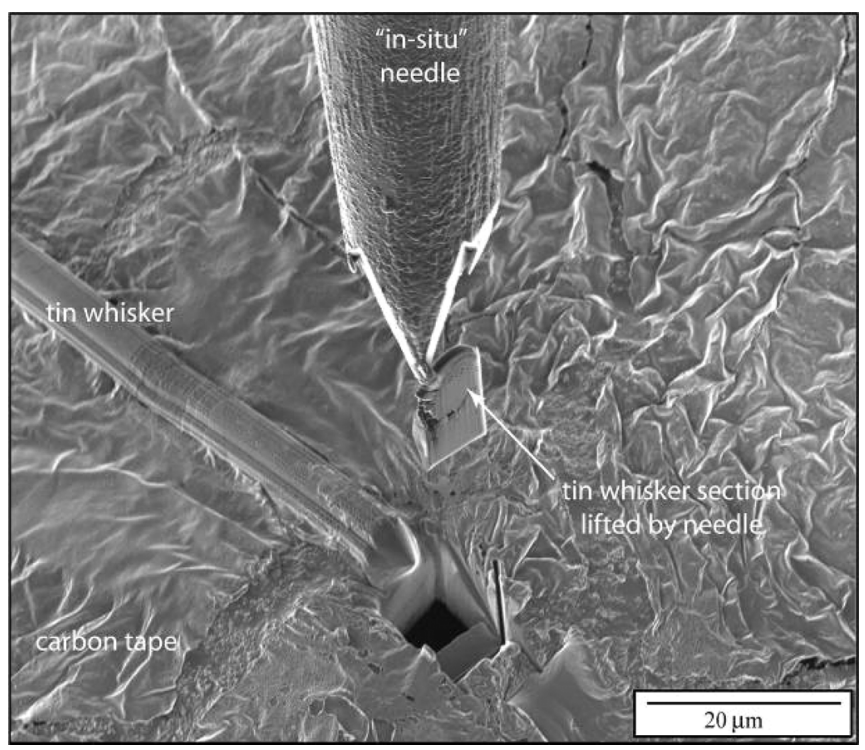

Fig. 6. FIB image showing removal of tin whisker section using the in situ needle (NASA/UCF).

ATVC Serial Number 33. There are 28 card guides from each LRU; however, card guides that had been used for other experiments were removed from selection. Ten card guides were randomly selected from each LRU and five whiskers were tested from the top, and five whiskers were tested from the bottom of each card guide, resulting in a sample size of 200 whiskers.

\section{Sample Preparation for Transmission Electron Microscopy (TEM)}

An in situ focused ion beam (FIB) methodology was used to prepare a tin whisker cross-sectional sample for TEM examination. The first step was to deposit platinum on top of the region of interest (ROI) along the whisker to protect the whisker outer surface from the ion beam. Next, the ion beam was used to mill trenches on either side of the ROI of the whisker normal to the direction of growth leaving a small $(<1 \mu \mathrm{m})$ section of the whisker.

Once the section was milled to a desired thickness, an in situ needle was lowered in preparation for removing the tin whisker section. The needle was spot welded to the whisker section using platinum, and the section was released from the carbon tape by milling away any remaining point of attachment with the FIB, as shown in Fig. 6.

A conventional TEM copper grid was sectioned, and a small ditch was made on the grid inner surface with the FIB to accommodate the ROI. The tin whisker section was lowered and inserted in the copper grid in preparation for TEM examination. The section was first spot welded to the grid, and then released from the in situ needle. The last step prior to placing the grid and sample into the TEM was to thin out the sample using the FIB until a thickness of $<1000 \AA$ was achieved. The TEM images and diffraction patterns were taken using a FEI Tecani F30 TEM with a 30-kV field emission source. 


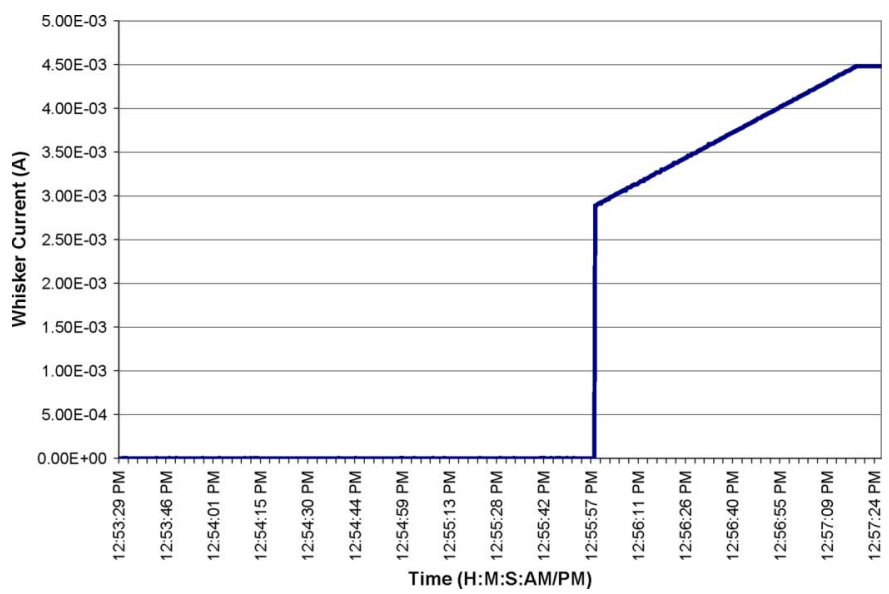

Fig. 7. Tin whisker No. 137 graph of current versus time from the second experiment.

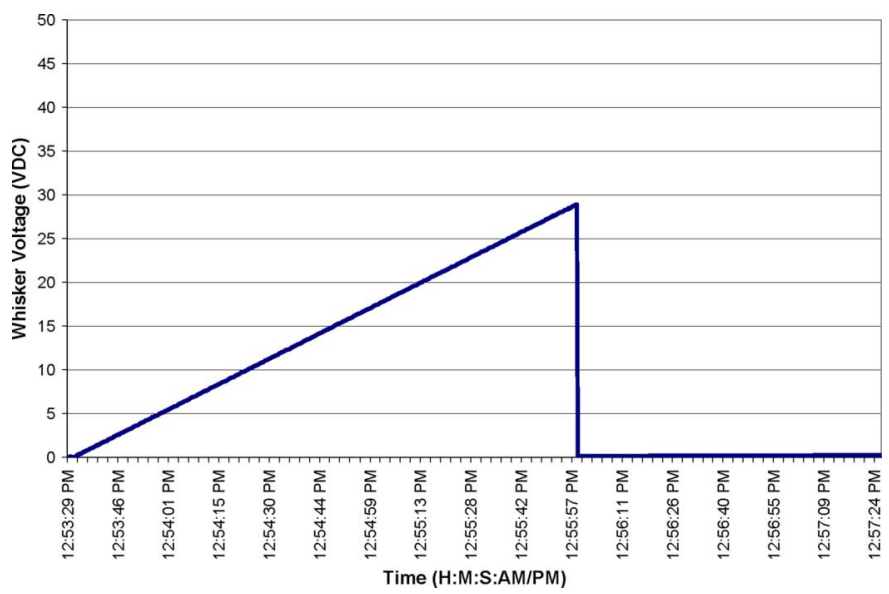

Fig. 8. Tin whisker No. 137 graph of voltage versus time from the second experiment.

\section{EXPERIMENTAL RESULTS}

\section{A. Tin Whisker Breakdown Voltage}

The point at which a short occurs, when the film resistance breaks down, can easily be seen in Fig. 7 when the current jumps from near zero, the nanoamp range, to the milliamp range. Prior to breakdown the majority of the voltage drop is across the whisker due to the high resistance of the oxide film on the whisker. In this state, the whisker voltage reading tracks close to the power supply voltage. The power supply voltage increases linearly from 0 to $45 \mathrm{Vdc}$, then it remains at $45 \mathrm{Vdc}$ for a few seconds at the end of the run until the software is given a stop command. After the film has broken down, the majority of the voltage drop is across the current limiting resistor. In this state, the low whisker voltage reading was determined by the small resistance of the whisker, card guide and micromanipulator as shown in Fig. 8.

The voltage level at the transition to metallic conduction current is the voltage level at which the film and oxide layers break down. Reference Fig. 8. As in the first experiment, the graphs of voltage and current data showed single transitions, multiple transitions, and multiple transitions with intermittent contact.

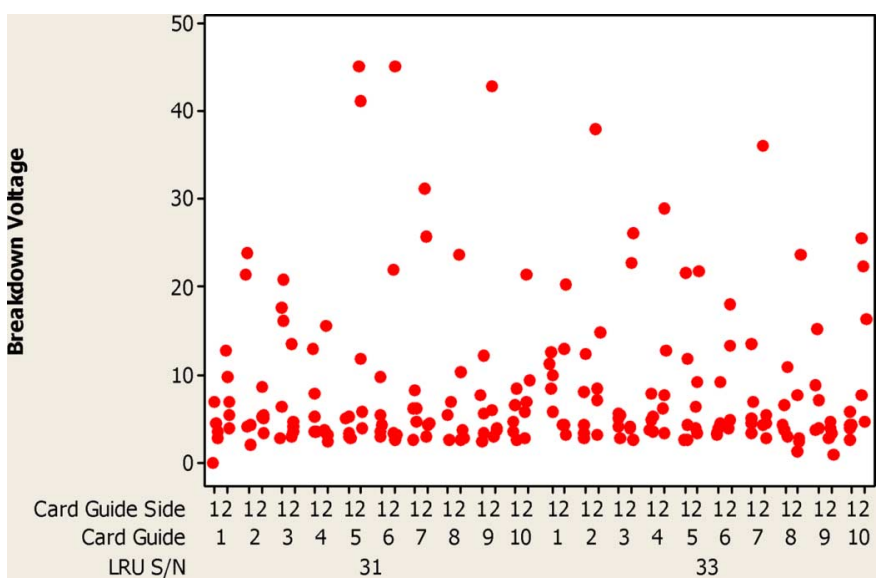

Fig. 9. Breakdown voltage individual value plot.

The breakdown voltage for each of the 200 tin whiskers is displayed in Fig. 9. The breakdown voltage for each of the whiskers was selected first by visual review of the graphs as was done in the first experiment. To ensure that a more consistent approach was used in the data collection process in the second experiment, a computer-based method for selecting the breakdown voltage was developed using Microsoft Excel. All 200 breakdown voltages were verified using both methods.

Since whiskers number 49 and 56 did not breakdown in the 0 to $45 \mathrm{Vdc}$ range used in this experiment, these two data points are considered censored. Minitab [9] was used in the second experiment because it contained a feature to easily accommodate censored data.

\section{B. Data Analysis}

The individual value plot shown in Fig. 9 provides insight into the data. The majority of the breakdown voltage values fall between 0 and $10 \mathrm{Vdc}$. The aforementioned figure shows tin whisker breakdown voltages versus card guide side where $1=$ top and $2=$ bottom, card guide, and LRU serial number. The right skewed nature of the data is evident from this plot. The individual values are shown in red.

By default, Minitab explores the following 11 distributions for the best fit: Weibull, Lognormal, Exponential, Loglogistic, 3-Parameter Weibull, 3-Parameter Lognormal, 2-Parameter Exponential, 3-Parameter Loglogistic, Smallest Extreme Value, Normal, and Logistic.

In the first experiment, we used the Probability-Probability (P-P) plots along with the Kolmogorov-Smirnov test to determine the best fitting distribution. Since Minitab does not perform the Kolmogorov-Smirnov test, the adjusted Anderson-Darling test and correlation coefficient were used in its place, in addition to the P-P plots [4]. The adjusted Anderson-Darling test measures the area between the fitted line (based on chosen model) and the nonparametric step function (based on the plot points) [10].

The correlation coefficient reported for each graph (distribution) measures the correlation between the data and what would be expected if the data comes from the specified distribution (represented by a line). If the data and the line are perfectly correlated, the correlation coefficient would be 1 , and that would 


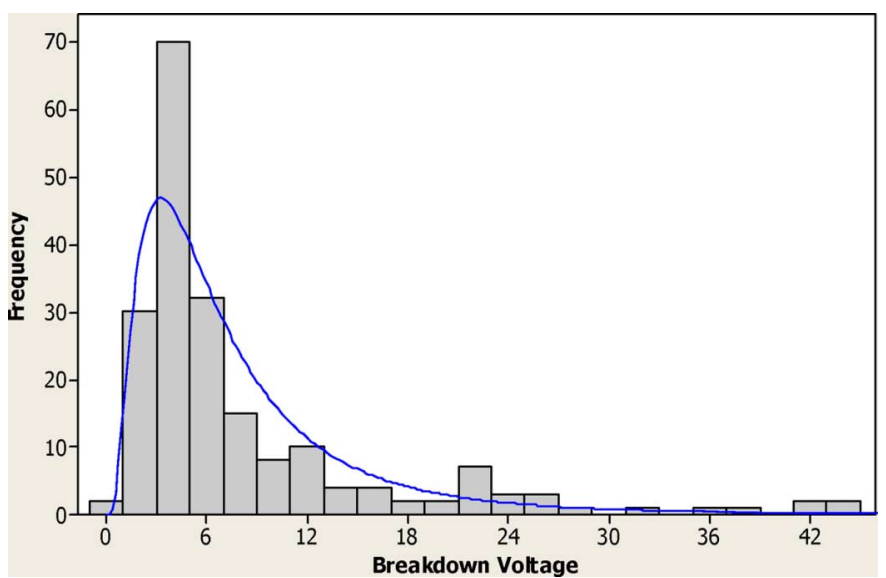

Fig. 10. Histogram tin whisker breakdown voltages with pdf of lognormal distribution.

strongly indicate that the data probably came from the specified distribution. Note that even if one generated the data from a given distribution, typically the correlation coefficient would not equal to 1 due to random variability. However, larger correlation coefficients indicate that the "fit" of the probability plot is better. The correlation coefficient for the Lognormal distribution was 0.943 .

Based on the P-P plots and the correlation coefficients, the Anderson-Darling (adjusted) test, the applicability of the distributions, and the principle of parsimony the Lognormal distribution was chosen as the best fitting model for the data

The pdf for the Lognormal distribution is shown in (1.3) below [10] as

$$
f(x)=\frac{1}{\sigma x \sqrt{2 \pi}} \exp \left(-\frac{(\ln (x)-\mu)^{2}}{2 \sigma^{2}}\right) .
$$

From the data, the estimated of the location parameter $=$ $\mu=1.77895$, and the scale parameter $=\sigma=0.776320$ were obtained. The pdf along with a histogram of the breakdown voltage data is shown in Fig. 10.

Based on the data and the fitted model, the expected (mean) voltage where a short will occur is $8.0067 \mathrm{Vdc}$, with a standard deviation of $7.2812 \mathrm{Vdc}$. The median tin whisker breakdown voltage is $5.9236 \mathrm{Vdc}$.

\section{Comparison of First and Second Experiment Results}

The empirical probability distributions derived from the data gathered in the first and the second experiments were the 3P-IG and the Lognormal distributions, respectively. It is important to note that EasyFit was used for fitting the distribution in the first experiment, and Minitab was used for fitting the distribution in the second experiment. Minitab contained a feature to easily handle censored data. The IG distribution is not evaluated by Minitab, and thus was not a choice in the second experiment. Takagi noted that the probability density functions of the IG and the Lognormal distributions are similar in shape [10]. The probability density functions for both experiments are right skewed. The larger sample size in the second experiment likely results in a better estimate of the tail of the distribution.
To aid in comparing the results of the second experiment to first experiment, the data from the first experiment was analyzed using Minitab. Based on the P-P plots, adjusted Anderson-Darling test, and the correlation coefficient, the Lognormal was the best fitting distribution using Minitab. From this analysis, it was evident that the first and second experiments are consistent when using the same software for fitting the distributions.

Based on the data and the fitted model for the first experiment, the expected voltage (mean) where a short will occur is $15.5994 \mathrm{Vdc}$, and the median tin whisker breakdown voltage is $11.8924 \mathrm{Vdc}$. Based on the data and the fitted model for the second experiment, the expected voltage (mean) where a short will occur is $8.0067 \mathrm{Vdc}$, and the median tin whisker breakdown voltage is $5.9236 \mathrm{Vdc}$. The shift in the mean can be explained partially by the change to a gold-plated probe tip in the second experiment, thus eliminating any effect of oxides on the probe tip.

\section{Tin Whisker Current Carrying Characteristics}

Since the power supply was limited to $45 \mathrm{Vdc}$ in this experiment, and the current limiting resistor was $10 \mathrm{k} \Omega$, the current through the whisker was limited to $4.5 \mathrm{~mA} .158$ out of 200 whiskers or $79 \%$ (95\% Confidence Interval $(72.69 \%, 84.43 \%)$ ) of the whiskers were able to carry $4.5 \mathrm{~mA}$. In some circuits, this current-carrying capability is enough to cause permanent short circuits.

\section{E. Limitations}

Two whiskers in the second experiment did not experience a breakdown of the film resistance in the $0-45 \mathrm{Vdc}$ range of the experiment. This resulted in two censored values out of the 200 whiskers tested. Increasing the upper voltage limit of the power supply voltage could eliminate the censoring.

The difference and variation between force applied by gravity and the force applied by the micromanipulator probe was another limitation. To improve control of the applied pressure in the second experiment, the probe was applied to the whisker on approximately the top $25 \%$ of the whisker. This minimizes the applied pressure, but does not completely eliminate the difference.

Another limitation of this experiment is the number of conducting surfaces. A free whisker falling across two contacts will have two points of contact for breakdown, while the micromanipulator probe contacts the whisker at one point. This was accepted simplification in this experiment.

To simulate a loose whisker bridging electrical contacts, all of the testing done in this experiment was performed by bringing the probe in contact with the side of a whisker. However, whiskers can also cause short circuits when a whisker grows from one lead and the tip of the whisker contacts an adjacent lead. In this case, with tip contact, the results may be different from those obtained with side contact due to contact area and force.

\section{F. Transmission Electron Microscopy (TEM)}

During the preliminary tin whisker characterization in the first experiment, FIB analysis and ion channeling imaging re- 


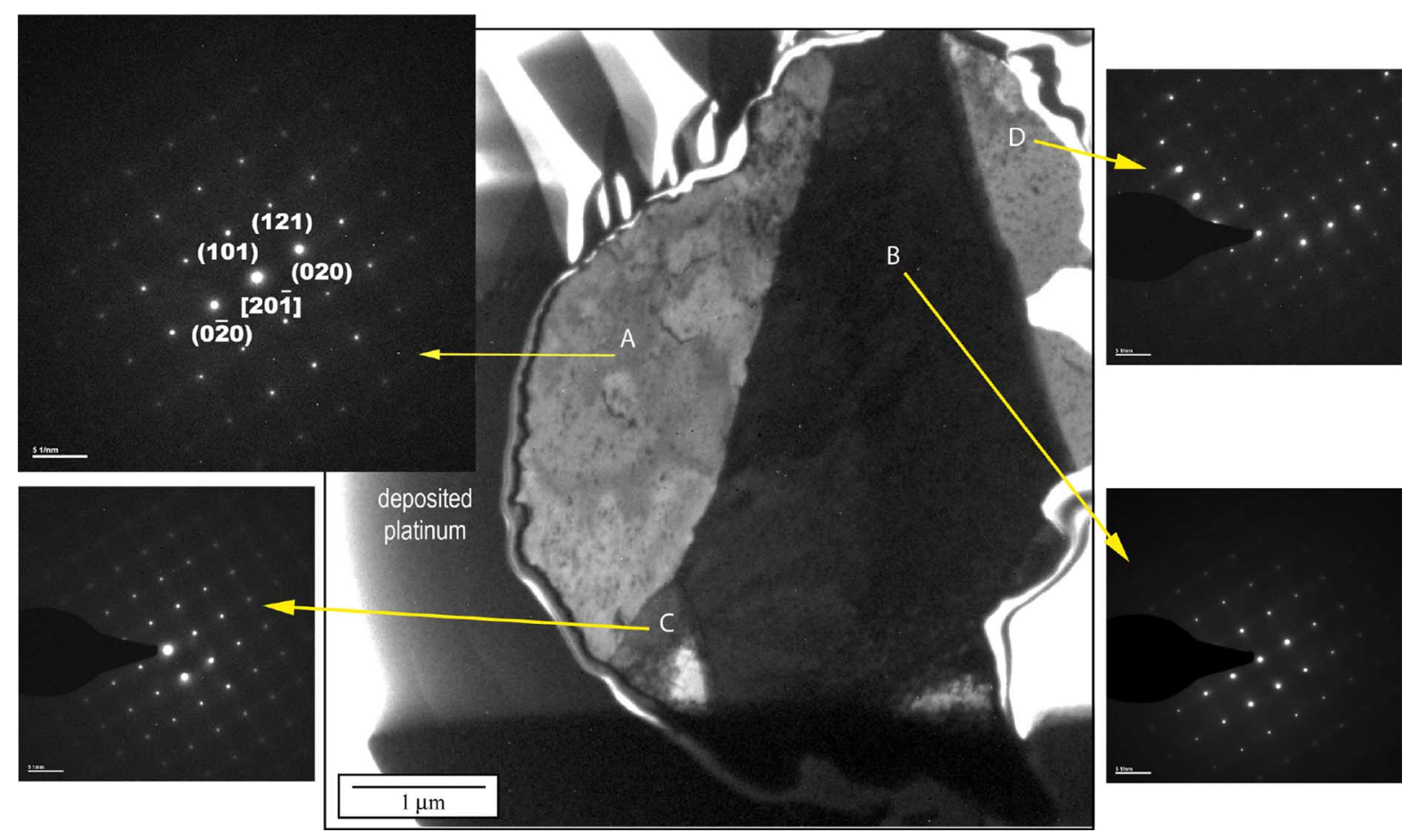

Fig. 11. Bright field TEM image of the polycrystalline tin whisker and nomenclature used to identify the various regions (A-D). Regions A-C were nearly identical with one another, while region $D$ was misoriented by approximately $2^{\circ}$ with region A (NASA/UCF).

vealed what appeared to be a polycrystalline whisker (refer to Fig. 1). In order to determine whether the whisker was polycrystalline, a thin section was prepared for TEM analysis as shown in Fig. 11. This sample is from a different section of the same tin whisker shown in the Fig. 1, but is rotated as evident by the location of the deposited platinum layer. The selected area diffraction patterns (SADPs) were taken at four site specific regions, labeled A, B, C, and D as shown in Fig. 11.

The SADPs obtained from regions A-D indexed to the tetragonal crystal structure of tin in the [201] beam direction (refer to Fig. 11). Region D was misoriented approximately $2^{\circ}$ with respect to region A in the (121) direction. Regions A-C were nearly identical with one another.

High-resolution TEM imaging, shown in Fig. 12, was used to image an amorphous region between uniform crystal lattices of regions $\mathrm{A}$ and $\mathrm{B}$, which clearly delineates a grain boundary between the crystals in the polycrystalline tin whisker. Additionally, X-ray energy dispersive spectroscopy (EDS) was used to verify that there were no compositional differences between the regions, all were composed of pure tin $(\mathrm{Sn})$. The polycrystalline structure of the studied whisker is shown by the contrast in regions A-D in the bright field TEM image in Fig. 11, the misorientation of region $\mathrm{D}$ with respect to region $\mathrm{A}$ shown in the SADPs, and the amorphous region between the crystals in the high-resolution TEM image in Fig. 12.

\section{G. Card Guide Cross Sections Using a FIB}

FIB analysis of two card guides was used to determine the grain size and thickness of the tin plating. Ion channel imaging was used to acquire images showing distinct grains based on

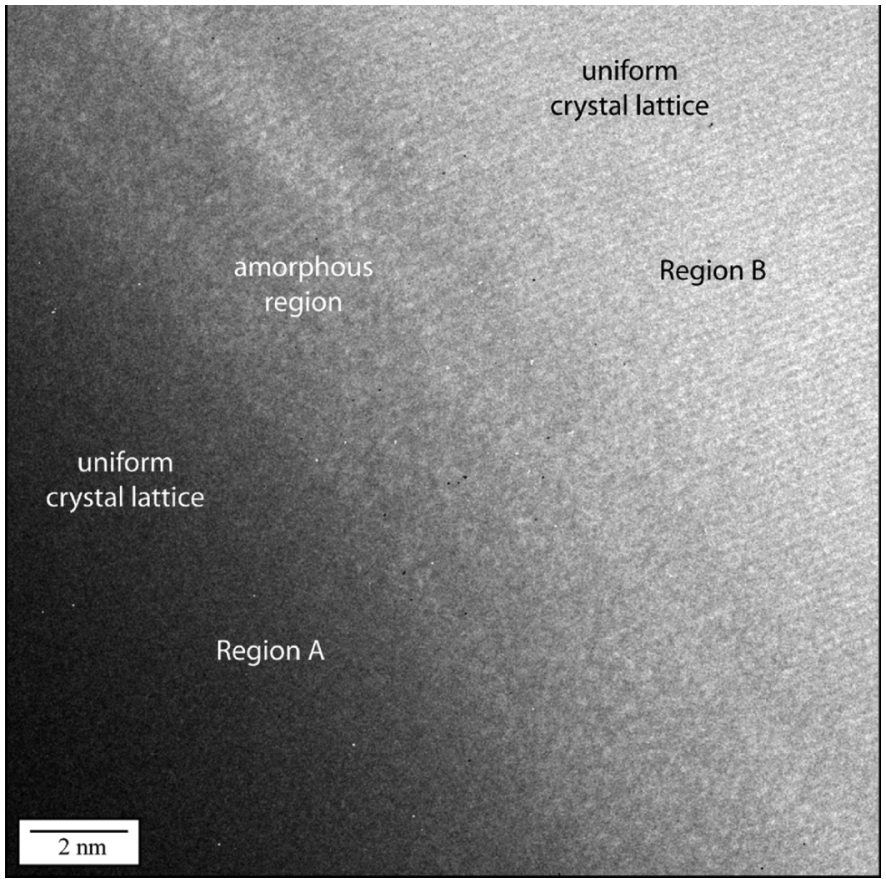

Fig. 12. High-resolution TEM image of the amorphous region in the polycrystalline tin whisker between the uniform crystal lattices of regions $\mathrm{A}$ and $\mathrm{B}$. The amorphous region is a low-angle grain boundary (NASA/UCF).

crystal orientation contrast as shown in Fig. 13. Using a modified line-intercept method, the average grain size for the card guide from ATVC S/N 31 shown in Fig. 13 was estimated to be $0.350 \mu \mathrm{m}(350 \mathrm{~nm})$, and the average grain size for the card guide from ATVC S/N 33 was estimated to be $0.290 \mu \mathrm{m}(290 \mathrm{~nm})$, 


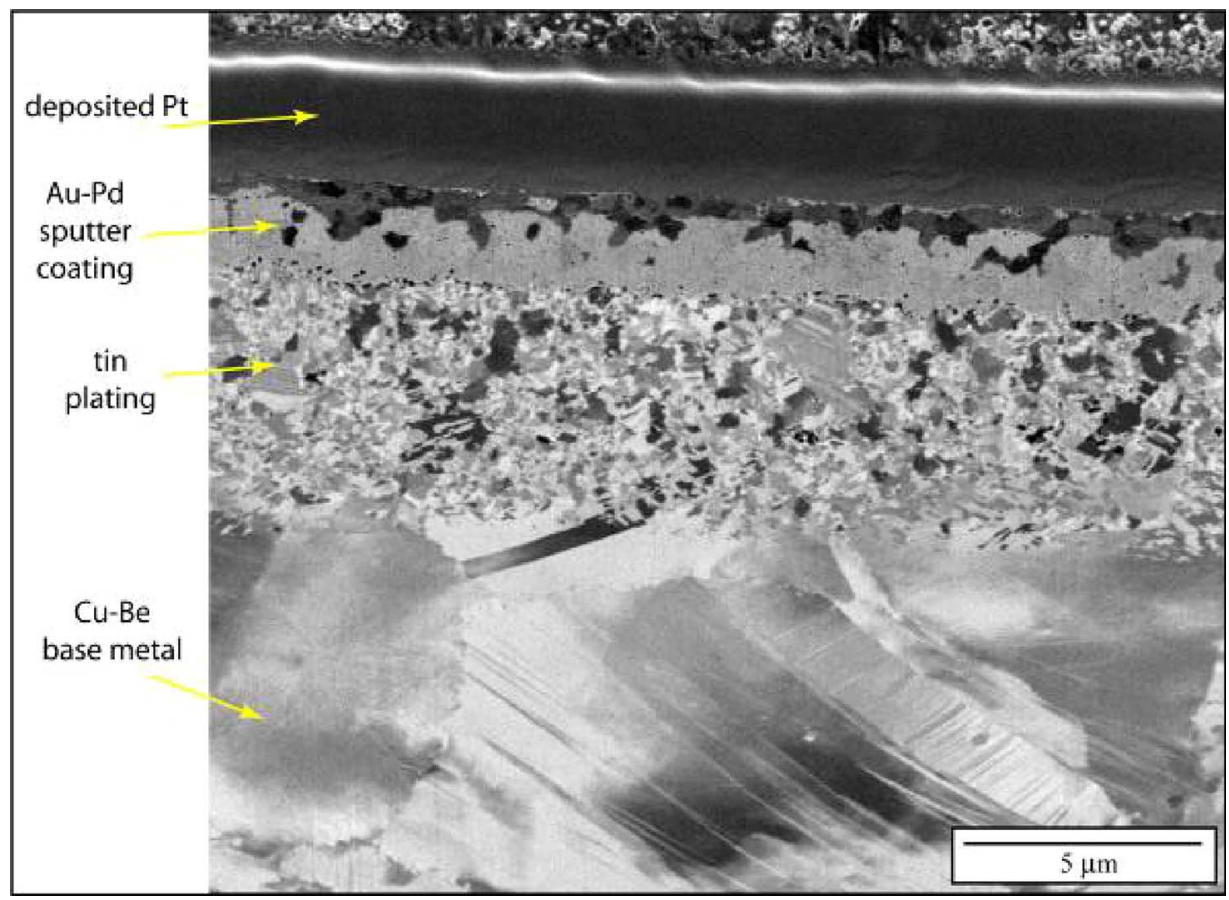

Fig. 13. FIB ion channeling image of card guide 16 (ATVC S/N 31) cross section showing the distinct layers studied: the expected Cu-Be substrate with $\mu \mathrm{m}$-sized grains, the Sn plating with nm-sized grains, the Au-Pd sputter coating and finally the deposited Pt used to protect the region during FIB analysis (NASA/UCF).

which falls well below the lowest grain size in the ASTM grain size number charts (grain size number $\gg 14$ ) [11]

Fig. 13 shows the copper-beryllium $(\mathrm{Cu}-\mathrm{Be})$ substrate base metal and the tin Sn plating. A layer of gold-palladium (Au-Pd) was sputter coated on top of the tin plating prior to the FIB sectioning, and a layer of platinum $(\mathrm{Pt})$ was deposited along the region of interest in the FIB to protect the sample during ion beam milling. The average thicknesses of the Sn layers are $6.9 \mu \mathrm{m}$ and $2.7 \mu \mathrm{m}$ for the card guides from ATVC S/N 31 and 33, respectively. EDS in a field-emission SEM was used to confirm the composition of each layer. The presence of beryllium cannot be confirmed using this technique since EDS will only accurately detect elements with a higher atomic number than Boron. However, the original drawings show the base metal for the card guide as copper-beryllium.

The purpose of measuring the grain size was to quantitatively determine the finish of the tin plating. Shetty classified large grain matte finish as having a grain size between 3-8 $\mu \mathrm{m}$, fine grain matte finish as having a grain size between 1-2 $\mu \mathrm{m}$, and bright finish as having a grain size $<1 \mu \mathrm{m}$ [12] . Based on these criteria, the tin plating used in both ATVC S/N 31 and 33 can be classified as bright finish. While tin finish was not a variable in this experiment, it is a point of interest because bright tin finishes have been associated with greater tin whisker growth than matte tin finishes [13], [14].

\section{CONCLUSION}

An empirical model to quantify the probability of occurrence of an electrical short circuit from tin whiskers as a function of voltage was developed in the first experiment [4]. It provided insight into the right skewed shape of the probability model.
The 3P-Inverse Gaussian distribution was used to describe the whisker breakdown voltage in this experiment. This probability model also provided the baseline for a simulation to evaluate the sample size for the second experiment. In the first experiment, a sample size of 35 tin whiskers was used. In the second experiment, a sample size of 200 tin whiskers was used to improve the accuracy of the probability model. The Lognormal distribution was found to be the best fitting distribution to describe the whisker breakdown voltage in the second experiment.

The lessons learned from first experiment also aided in improving the equipment and procedures for the second experiment. The modified solder's helper, extension platform, and the ferrous top plate greatly improved the ability to clearly see and probe the whiskers under a microscope.

Three tin whiskers were cross sectioned using an FIB for study. Two of the whiskers exhibited the commonly reported single crystal structure. One whisker showed apparent variation in grain orientation within the cross section. Further examination was performed using a TEM. High-resolution TEM imaging was used to examine an amorphous region between uniform crystal lattices. This clearly delineates a grain boundary between the crystals in the polycrystalline tin whisker. SADPs indicated a $2^{\circ}$ misorientation between two regions. The polycrystalline structure of the tin whisker is shown by the TEM images and the SADPs.

In addition, since bright tin finishes have been associated with greater tin whisker growth than matte tin finishes, two card samples were prepared, one from each LRU, and were sectioned using a FIB. Using a modified line-intercept method, the average grain size for the card guides' tin finish was determined to be in the nm-range, indicative of a bright finish. 


\section{A. Future Work}

There are many aspects of the tin whisker phenomena that provide opportunities for future research. One area that would expand on the research performed here would be to study the effect of pressure on breakdown voltage. This would require the development of a method measuring pressure applied to the tin whisker either directly or indirectly by measuring whisker deflection, length and diameter and then calculating the pressure.

Expanding the range of the power beyond the $0-45 \mathrm{Vdc}$ to determine the upper limit of breakdown voltage would also provide additional insight. By expanding the upper limit one may be able to eliminate the censored data points.

Other variables that could affect tin whisker shorting should also be studied. Some examples of these variables that warrant future study include whisker shape, whisker length and thickness, and oxidation layer thickness. Another phenomenon related to tin whiskers that warrants further quantification is metal vapor arcing.

\section{ACKNOWLEDGMENT}

The authors would like to thank Dr. H. Leidecker of NASA and J. Brusse of Perot Systems at Goddard Space Flight Center for sharing their vast knowledge on the topic of tin whiskers, and taking the time to answer the many questions posed throughout this experiment. The authors would also like to thank Z. Rahman with the Materials Characterization Facility, AMPAC, University of Central Florida (UCF), for his expertise in FIB and TEM analysis, M. Spates, P. Marciniak, S. Loucks, J. Neihoff, P. Richiuso, and R. King of NASA Kennedy Space Center for their help with the fabrication/modification of the test equipment, L. Batterson of NASA Kennedy Space Center for his expertise in photography, A. Oliu of NASA Johnson Space Center for his expertise with digital imaging, Dr. L. Keller of NASA Johnson Space Center and Dr. J. Lomness of NASA Kennedy Space Center for reviewing the diffraction patterns, Dr. S. Smith of NASA and Dr. R. N. Shenoy of Lockheed Martin at NASA Langley Research Center for indexing the diffraction patterns, and M. Madden of United Space Alliance for his expertise with breakdown voltage selection software.

\section{REFERENCES}

[1] G. T. Galyon, "Annotated tin whisker bibliography and anthology," IEEE Trans. Electron. Packag. Manuf., vol. 28, no. 1, pp. 94-122, Jan. 2005.

[2] J. Brusse, G. Ewell, and J. Siplon, "Tin whiskers: Attributes and mitigation," in Proc. Capacitor and Resistor Technol. Symp., 2002, pp. 67-80.

[3] H. Leidecker and J. Brusse, "Tin whiskers: A history of documented electrical system failures-A briefing," Space Shuttle Program Office, 2006 [Online]. Available: http://nepp.nasa.gov/whisker/reference/tech_papers/2006-Leidecker-Tin-Whisker-Failures.pdf.

[4] K. J. Courey, S. S. Asfour, J. A. Bayliss, L. L. Ludwig, and M. C. Zapata, "Tin whisker electrical short circuit characteristics-Part I," IEEE Trans. Electron. Packag. Manuf., vol. 31, no. 1, pp. 32-40, Jan. 2008.

[5] T. Fang, "Tin whisker risk assessment studies," DAI-B, 2005, vol. 66(12) [Online]. Available: https://drum.umd.edu/dspace/handle/ $1903 / 3079$

[6] R. D. Hilty and N. E. Corman, "Tin whisker reliability assessment by Monte Carlo simulation," in Proc. IPC/JEDEC Lead-Free Symp., 2005, pp. 1-8.

[7] EasyFit. [Electronic] Mathwave Technologies, 2007, vol. 3.2 [Online]. Available: http://www.mathwave.com/products/easyfit.html
[8] I. A. Koutrouvelis, G. C. Canavos, and S. G. Meintanis, "Estimation in the three-parameter inverse Gaussian distribution," in Comput. Statist. Data Anal., Jun. 15, 2005, vol. 49, pp. 1132-1147.

[9] "Minitab Release 14 Statistical Software," Minitab Inc., 2003 [Online]. Available: http://www.minitab.com/products/

[10] K. Takagi, S. Kumagai, C. Matsunaga, and Y. Kusaka, "Application of inverse Gaussian distribution to occupational exposure data," The Annals of Occupational Hygiene, vol. 41, pp. 505-514, Oct. 1997.

[11] "Standard Test Methods for Determining Average Grain Size," American Society for Testing and Materials, ASTM International, West Conshohocken, PA, 2006 [Online]. Available: www.astm.org.

[12] R. Schetty, "Electrodeposited tin properties \& their effect on component finish reliability," in Proc. 2004 Int. Conf. Business of Electron. Product Reliability and Liability, 2004, pp. 29-34.

[13] J. Smetana, "Minimizing tin whiskers," SMT Surface Mount Technol. Mag., vol. 19, pp. 36-38, 2005.

[14] M. Osterman, in Proc. Mitigation Strategies for Tin Whiskers, Jul. 3, 2002, vol. 2006.

Karim J. Courey received the B.E.E. degree from Cleveland State University, Cleveland, OH, in 1986, the M.B.A. degree from the Florida Institute of Technology, Melbourne, FL, in 1993, and the Ph.D. degree in industrial engineering from the University of Miami, Coral Gables, FL, in 2008.

He is currently a Principal Engineer with the Orbiter Sustaining Engineering Office for the National Aeronautics and Space Administration, Lyndon B. Johnson Space Center, Texas. His duty location is at the John F. Kennedy Space Center in Florida.

Dr. Courey is a licensed Professional Engineer in the state of Texas.

Shihab S. Asfour received the B.S. and M.S. degrees in production engineering from Alexandria University, Alexandria, Egypt, in 1973 and 1976, respectively, and the Ph.D. degree in industrial engineering and psychology from Texas Tech University, Lubbock, in 1980.

He is currently Professor and Associate Dean of the College of Engineering at the University of Miami, Coral Gables, FL. He has over 30 years of instructional, professional and research experience and has authored over 200 publications. He has 17 recent publications in both biomechanical and industrial engineering and has also supervised $18 \mathrm{Ph} . \mathrm{D}$. dissertations.

Arzu Onar received the B.S. degree in mathematical sciences at the University of North Carolina, Chapel Hill, in 1993 and the M.S. and Ph.D. degrees in statistics from University of South Carolina, Columbia, in 1995 and 1998, respectively.

She is currently a faculty member at the Biostatistics Department, St. Jude Children's Research Hospital, Memphis, TN. Her research interests are in statistical reliability and in survival analysis.

Jon A. Bayliss received the A.S. degree in computer engineering technology from Tampa Technical Institute, Tampa, FL, in 1979, and the B.S. degree in electrical engineering from the Florida Institute of Technology, Melbourne, in 1986.

He is currently an Electrical Engineer in the Electronic Failure Analysis Section, Engineering Directorate for the National Aeronautics and Space Administration, Kennedy Space Center, FL.

Lawrence L. Ludwig received the B.S. degree in electrical engineering from the Florida Institute of Technology, Melbourne, in 1986 and the M.S. degree in engineering management from the University of Central Florida, Orlando, in 1992.

He is currently a Lead Electrical Engineer in Electronic Failure Analysis Section, Engineering Directorate for the National Aeronautics and Space Administration, Kennedy Space Center, FL.

Maria C. Wright received the B.S. degree in materials science and engineering from the University of Florida, Gainesville, in 2002 and the M.S. degree in materials science and engineering from the Pennsylvania State University, University Park, in 2005.

She is currently a Materials Engineer in the Materials Failure Analysis Section, Engineering Directorate for the National Aeronautics and Space Administration at Kennedy Space Center, FL. 